5. G. J. Swanson and W. B. Veldkamp, "Diffractive optical elements for use in infrared systems," Opt. Eng. 28, 605-608 (1989).

6. J. R. Leger, M. L. Scott, P. Bundman, and M. P. Griswold, "Astigmatic wavefront correction of gain-guided laser diode array using anamorphic diffractive micro-lenses," in Computer
Generated Holography II, S. H. Lee, ed., Proc. Soc. Photo-Opt. Instrum. Eng., 884, 82-89 (1988).

7. K. S. Urquhart and H. Farhoosh, "Diffractive lenses using orthogonal cylindrical Fresnel zone plates," in Computer and Optically Formed Holographic Optics, I. Cindrich and S. H. Lee, eds., Proc. Soc. Photo-Opt. Instrum. Eng. 1211, 184-190 (1990).

\section{Analysis of the holographic reciprocity law for dichromated gelatin}

\section{Pascual, A. Beléndez, and A. Fimia}

The authors are with the Departamento Interuniversitario de Optica, Laboratorio de Optica, Universidad de Alicante, Apartado 99, Alicante E 03080, Spain.

Received 30 September 1991.

0003-6935/92/173200-02\$05.00/0.

(c) 1992 Optical Society of America.

The holographic reciprocity law for dichromated gelatin is investigated experimentally. The results obtained show that this law does not hold true for this material.

Key words: Holography, recording materials, reciprocity law.

The reciprocity law for a holographic recording material is a function of the total exposure $E=I \times t$ only and is independent of the magnitude of either irradiance $I$ or exposure time $t .{ }^{1}$ However, we see below that the reciprocity law does not hold true for exposures of high irradiance (short duration) and for exposures of low irradiance (long duration). This phenomenon is called reciprocity law failure. Several authors have reported a reciprocity law failure for silver halide emulsions. ${ }^{1,2}$ For dichromated gelatin (DCG) Chang and Leonard found a significant change in holographic characteristics that were due to the effects of reciprocity. ${ }^{3}$

DCG has high energy sensitivity in a spectral region in which no laser sources can be found, which makes it impossible to use the chromatic sensitivity of this recording material. Thus it is necessary to expose the plate of DCG for long periods of times. If we work with short wavelengths, in which DCG has more sensitivity, it is possible to prove that the DCG response is different because its diffraction efficiency for a fixed level of exposure has been modified.

The effects of the reciprocity law on DCG were investigated by recording transmission holographic gratings at the same value of exposure but with different irradiances. Measurements of diffraction efficiency for these gratings

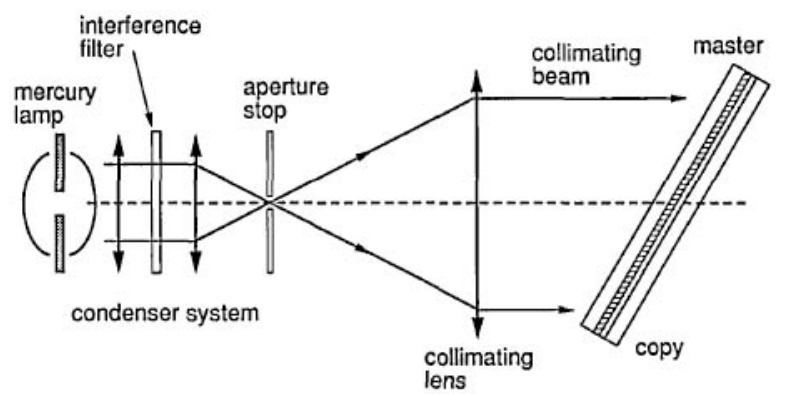

Fig. 1. Experimental setup used to record transmission holographic gratings in DCG. indicate that efficiency depends on the irradiance for a fixed level of exposure.

Experiments were carried out with DCG obtained from Kodak $649 \mathrm{~F}$ plates. In order to obtain holographic gratings in DCG we used a two-step method for recording holographic optical elements with partially coherent light. ${ }^{4}$ The method consists of copying a previously generated holographic grating in Fresnel's region. When the grating is placed at Bragg's angle and its object beam and reference beam in reconstruction are optimized so that they have a 1 to 1 relation in the second stage, we are able to copy onto a DCG plate. The diffraction efficiency of the second holographic grating is comparable to that obtained by using a conventional holographic device.

Figure 1 shows the experimental setup. A collimated beam from a high-pressure mercury lamp was used to copy a series of transmission gratings with exposures ranging from 1.5 to $125 \mathrm{~mJ} / \mathrm{cm}^{2}$. The coherence factor of this light is more than 0.9 . The sensitivity of DCG increases by a factor of 100 when we tune from 514 to $405 \mathrm{~nm}$ (see Ref. 5) so we chose $405 \mathrm{~nm}$ as a recording wavelength.

After exposure the plates were developed in a DCG standard developing process. It is possible to compare the results obtained by this method with the results we see reported in the literature (see Ref. 6) that use the same recording material and process we use with coherent sources.

The results are summarized in Figs. 2 and 3, which show the diffraction efficiency versus exposure for three irradiance sources, and the diffraction efficiency versus the exposure time, also for three irradiance sources.

As can be seen from Fig. 2 the exposure that allows us to obtain maximum diffraction efficiency varies from 20 to 80 $\mathrm{mJ} / \mathrm{cm}^{2}$, depending on the source of irradiance. We can make an interesting observation: when the power of the source increases the sensitivity also increases. We think that an explanation of this increase of sensitivity in DCG is supported by the following argument. In DCG the photosen-

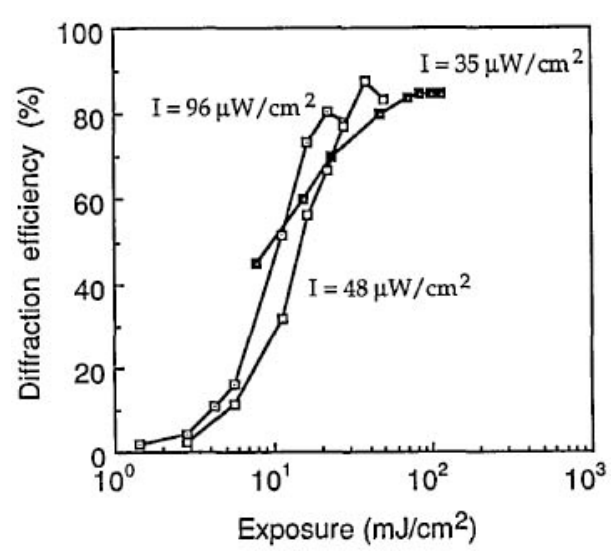

Fig. 2. Diffraction efficiency plotted as a function of exposure for three irradiance sources. 


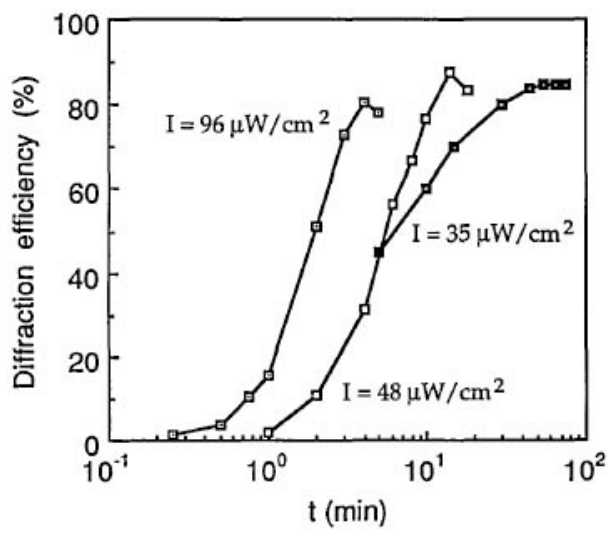

Fig. 3. Diffraction efficiency plotted as a function of the exposure time for three irradiance sources.

sitive components, that is, the dichromates, are efficient absorbers of ultraviolet, violet, and blue radiation. According to the reciprocity law in a photochemical reaction the yield of product is a function of the total radiant energy absorbed, i.e., exposure $E$. To obtain a given yield the required exposure time $t$ is inversely proportional to the irradiance $I$ that is used. This is because the quantum yield of the reduction of the dichromate to $\mathrm{Cr}^{+3}$ decreases for a prolonged exposure time. This quantum yield is the reciprocal of the number of quanta that are required for reducing one dichromate group. ${ }^{7}$
In summary, Figs. 2 and 3 show that, at equal exposure levels, the diffraction efficiency obtained for holographic gratings made in DCG is different. This clearly indicates an increase in the sensitivity of DCG and that a failure in the reciprocity law exists for this recording material. Finally, from these experiments we can also conclude that it is possible to increase the energetic sensitivity in DCG by increasing irradiance.

\section{References}

1. P. L. Bachman, "Silver halide photography," in Handbook of Optical Holography, H. J. Caufield, ed. (Academic, New York, 1979), pp. 110-112.

2. K. M. Johnson, L. Hesselink, and J. W. Goodman, "Holographic reciprocity law failure," Appl. Opt. 23, 218-227 (1984).

3. B. J. Chang and C. D. Leonard "Dichromated gelatin for the fabrication of holographic optical elements," Appl. Opt. 18, 2407-2417 (1979).

4. I. Pascual, A. Beléndez, and A. Fimia "A two step method for recording holographic optical elements with partially coherent light," J. Opt. (Paris) 22 135-140 (1990).

5. L. Solymar and D. J. Cooke, Volume Holography and Volume Gratings (Academic, London, 1981), pp. 278-286.

6. D. Meyerhofer, "Dichromated gelatin" in Holographic Recording Materials, Vol. 20 of Topics in Applied Physics, H. M. Smith, ed. (Springer-Verlag, Berlin, 1977), pp. 75-99.

7. J. Kosar, Light-Sensitive System: Chemistry and Application of Nonsilver Halide Photographic Processes (Wiley, New York, 1965), pp. 71-74.

\section{Phase shifting of whole-field fringes in dual-plate speckle photography}

\section{Guillermo H. Kaufmann and Pierre Jacquot}

G. H. Kaufmann is with the Applied Optics Group, Instituto de Fisica Rosario (CONICET-UNR), Rosario 2000, Argentina. P. Jacquot is with the Laboratory of Stress Analysis, Swiss Federal Institute of Technology, Lausanne CH-1015, Switzerland.

Received 25 February 1991.

0003-6935/92/173201-03\$05.00/0.

(1) 1992 Optical Society of America.

New developments of a phase-shifting readout system for the analysis of whole-field fringes in dual-plate speckle photography are presented.

Key words: Speckle photography, phase shifting, metrology.

A heterodyne readout of the fringes of dual-plate speckle photography is proposed by Smith and Stetson ${ }^{1}$ and Stetson $^{2}$ to overcome the practical limitations of the conventional double-exposure technique. In this readout scheme both specimen exposures are recorded on separate photographic plates and the two halos that are generated by pointwise filtering are combined in an interferometer. This technique has the advantage of permitting compensation for large specimen displacements between exposures. As it is based on direct local phase measurements it allows achieving subspeckle size accuracy. It is much less sensitive to the diffraction halo effect and also removes the sign ambiguity of the displacement components. However, exper- imental results show the appearance of important errors that are related to the simultaneous scanning of both photographic plates. ${ }^{3,4}$ Erratic tilts of any of the plate holders or unstable misalignments of the probing beams introduce spurious displacements that drastically reduce the measurement accuracy.

The drawbacks of this heterodyne technique have led us recently to propose a new readout approach. ${ }^{5}$ This new system is based on a combination of Fraunhofer diffraction patterns that are generated by both plates in a MachZehnder interferometer. By optically filtering both superimposed diffraction patterns whole-field fringes are formed. Speckle displacements are computed from the phase of these fringes by phase shifting one interferometer beam with respect to the other with a tilted glass plate. Even though preliminary results are promising, as with any other interferometric system, this readout scheme has from several important requirements. The alignment of mirrors, beam splitters, and both plates is a rather time-consuming process. For avoiding distortions in the speckle fringes the optical components must be of high quality. The system is also quite vulnerable to air turbulence and environmental vibrations, so tilting the glass plate for successive irradiance data acquisition must be performed rather quickly.

The purpose of this Note is to present a simpler phaseshifting readout scheme that offers substantial advantages over the previously developed scheme and can be easily automated. A schematic diagram of the readout system is shown in Fig. 1. Two specklegrams $P_{1}$ and $P_{2}$ are positioned in a specially designed plate holder that permits the translation of one of the glass plates over the surface of the other. Specklegram $\mathrm{P}_{1}$ can be translated in the $x$ and $y$ directions 